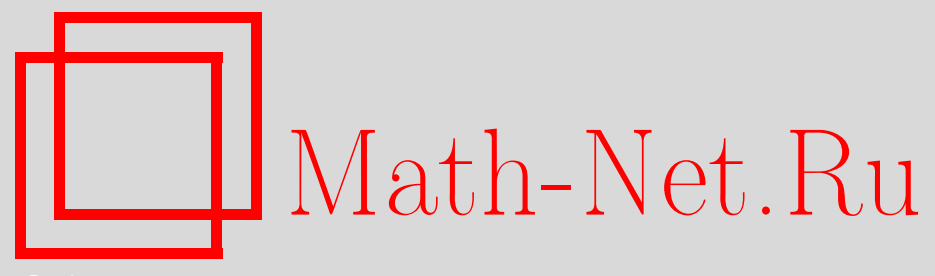

И. А. Круглов, Оценка скорости сходимости к равномерному распределению для произведений элементов конечной группы, управляемых цепью Маркова, Матем. вопр. криптогр., 2014, том 5, выпуск 1, 85-94

DOI: https://doi.org/10.4213/mvk108

Использование Общероссийского математического портала Math-Net.Ru подразумевает, что вы прочитали и согласны с пользовательским соглашением http: //www . mathnet.ru/rus/agreement

Параметры загрузки:

IP : 54.174 .149 .18

26 апреля 2023 г., 02:14:28

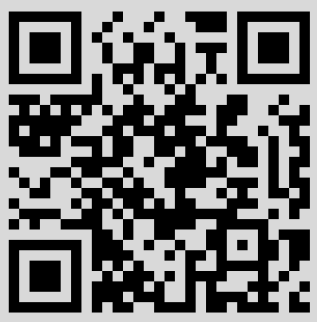




\title{
Оценка скорости сходимости к равномерному распределению для произведений элементов конечной группы, управляемых цепью Маркова
}

\author{
И. А. Круглов \\ ООО «Центр сертификационных исследований», Москва
}

Получено 22.IV.2013

Для произведений случайных подстановок, управляемых цепью Маркова, получена верхняя оценка среднеквадратического отклонения матриц вероятностей переходов, порожденных этими произведениями, от стохастической матрицы с одинаковыми элементами.

Ключевые слова: цепи Маркова, группы подстановок, матрицы вероятностей переходов

The estimate of the convergence rate to the uniform distribution for products of elements of finite group controlled by a Markov chain

\section{A. Kruglov}

\section{LLC "Sertification Research Center", Moscow}

Abstract. For products of random substitutions controlled by a Markov chain we obtain an upper bound for the mean-square deviation of transition matrices corresponding to these products from the stochastic matrix with equal elements.

Key words: Markov chain, substitution groups, transition probability matrices

Citation: Mathematical Aspects of Cryptography, 2014, vol. 5, no. 1, pp. 85-94 (Russian). 
Рассмотрим конечную простую однородную цепь Маркова

$$
\alpha_{1}, \alpha_{2}, \ldots, \alpha_{N}, \alpha_{N+1}, \ldots
$$

со множеством состояний $\{1,2, \ldots, n\}$, матрицей переходных вероятностей $P=[p(i, j)]_{i, j=1}^{n}$ и вектором начального распределения $\bar{p}_{0}$. Рассмотрим также произвольную конечную группу $G$ с групповой операцией «*» и предположим, что для любых $i, j \in\{1,2, \ldots, n\}$ задана последовательность $\left\{\xi_{i, j}^{(N)}\right\}_{N \geq 1}$ одинаково распределенных случайных элементов со значениями в группе $G$, распределения которых совпадают с распределением случайного элемента $\xi_{i, j}$, причем случайные элементы $\xi_{i, j}^{(N)}, N \geq 1, i, j \in\{1, \ldots, n\}$, независимы между собой и не зависят от цепи Маркова $\left\{\alpha_{N}\right\}_{N \geq 1}$. При любом $N \geq 1$ определим произведение случайных элементов

$$
\eta^{(N)}=\xi_{\alpha_{1}, \alpha_{2}}^{(1)} * \xi_{\alpha_{2}, \alpha_{3}}^{(2)} * \ldots * \xi_{\alpha_{N}, \alpha_{N+1}}^{(N)} .
$$

Пусть $q$ - произвольное подстановочное представление группы $G$ подстановками непустого конечного множества $S, m=|S|$ - степень представления $q$. Для любого случайного элемента $\xi$ со значениями в группе $G$ определена матрица переходных вероятностей (см. [1, § 1])

$$
\Pi_{\xi}^{(q)}=\left[p_{\xi}^{(q)}(\alpha, \beta)\right]_{\alpha, \beta \in S},
$$

где для любых $\alpha, \beta \in S$

$$
p_{\xi}^{(q)}(\alpha, \beta)=\sum_{\tau \in G_{\alpha, \beta}} \mathbf{P}(\xi=\tau), \quad G_{\alpha, \beta}=\left\{\tau \in G \mid \alpha^{q(\tau)}=\beta\right\} .
$$

Автором в $§ 3$ работы [1] было показано, что при выполнении широких условий последовательность матриц переходных вероятностей $\left\{\Pi_{\eta^{(N)}}^{(q)}\right\}_{N \geq 1}$ сходится при $N \rightarrow \infty$ к матрице $\left[\frac{1}{m}\right]$ размеров $m \times m$, все элементы которой равны $\frac{1}{m}$. При этом из доказательства следует, что в случае наличия такой сходимости для величин

$$
\varepsilon_{N}=\max _{\alpha \in S}\left(\sum_{\beta \in S}\left(p_{\xi}^{(q)}(\alpha, \beta)-\frac{1}{m}\right)^{2}\right)^{\frac{1}{2}}, \quad N \geq 1,
$$

справедлива экспоненциальная оценка порядка скорости сходимости к 0 при $N \rightarrow \infty$. Оценке порядка скорости сходимости величин $\varepsilon_{N}$ к нулю в одном 
частном случае посвящена также работа автора [2]. Однако ее результаты имеют лишь качественный характер.

В настоящей работе получена явная экспоненциальная оценка сверху для величины $\varepsilon_{N}$ в виде неравенства. Найдены дополнительные условия, при выполнении которых основание экспоненты строго меньше единицы. Основные из них состоят в том, что матрица $P$ переходных вероятностей цепи Маркова должна быть дважды стохастической и вполне неразложимой (т. е. при вычеркивании любой строки и любого столбца перманент получающейся матрицы должен быть положительным). Отметим, что впервые такие условия для доказательства сходимости к матрице вида $\left[\frac{1}{m}\right]$ (в том числе и для неоднородных цепей Маркова) были эффективно использованы В. Н. Сачковым [3].

Введем обозначения, необходимые для формулировки основного результата работы. Пусть для любого $\sigma \in G$ и любых $\alpha, \beta \in S$

$$
Q_{\alpha, \beta}(\sigma)= \begin{cases}1, & \alpha^{q(\sigma)}=\beta, \\ 0, & \alpha^{q(\sigma)} \neq \beta .\end{cases}
$$

Рассмотрим матричное представление $Q$ группы $G$, где

$$
Q(\sigma)=\left[Q_{\alpha, \beta}(\sigma)\right]_{\alpha, \beta \in S}, \quad \sigma \in G,
$$

соответствующее подстановочному представлению $q$. Выберем произвольное множество $\widehat{G}^{(q)}$ попарно неэквивалентных неприводимых унитарных матричных представлений группы $G$ над полем комплексных чисел, для которого любая неприводимая компонента представления $Q$ группы $G$ эквивалентна ровно одному представлению из множества $\widehat{G}^{(q)}$. Для любого случайного элемента $\xi$ со значениями в группе $G$ определена матричная характеристическая функция матрицы переходных вероятностей $\Pi_{\xi}^{(q)}$ (см. [1]):

$$
\Phi_{\xi}(U)=\sum_{\sigma \in G} \mathrm{P}(\xi=\sigma) U(\sigma), \quad U \in \widehat{\mathrm{G}}^{(q)} .
$$

Рассмотрим произвольное представление $U \in \widehat{G}^{(q)}$ степени $n_{U}$. Определим блочную квадратную матрицу

$$
\Phi(U)=\left[\begin{array}{cccc}
\Phi_{1,1}(U) & \Phi_{1,2}(U) & \ldots & \Phi_{1, n}(U) \\
\Phi_{2,1}(U) & \Phi_{2,2}(U) & \ldots & \Phi_{2, n}(U) \\
\vdots & \vdots & \vdots & \vdots \\
\Phi_{n, 1}(U) & \Phi_{n, 2}(U) & \ldots & \Phi_{n, n}(U)
\end{array}\right]
$$


порядка $n n_{U}$ с $n^{2}$ квадратными блоками порядка $n_{U}$, у которой для любых $i, j \in\{1, \ldots, n\}$ на месте $i, j$ стоит блок

$$
\Phi_{i, j}(U)=p(i, j) \Phi_{\xi_{i, j}}(U)
$$

Для произвольного вектора $\alpha$ унитарного пространства над полем комплексных чисел через $\|\alpha\|$ обозначим его евклидову норму, порожденную скалярным произведением в данном пространстве. При любом $U \in \widehat{G}^{(q)}$ обозначим через $\|\Phi(U)\|$ эрмитову норму матрицы $\Phi(U)$ как линейного преобразования унитарного пространства вектор-столбцов длины $n n_{U}$ над полем комплексных чисел. Пусть $U_{0} \in \widehat{G}^{(q)}-$ (одномерное) единичное представление группы $G$, определяемое соотношением

$$
U_{0}(g)=1 \quad \forall g \in G
$$

Положим

$$
\lambda=\max \left\{\|\Phi(U)\|: U \in \widehat{G}^{(q)}, U \neq U_{0}\right\} .
$$

Для произвольного случайного элемента $\xi$ со значениями в группе $G$ обозначим

$$
\Omega(\xi)=\{\sigma \in G: \mathbf{P}(\xi=\sigma)>0\}
$$

носитель его распределения. Рассмотрим множество $Z$ последовательностей вида

$$
\begin{gathered}
z=\left(j_{0}=1, i_{1}, j_{1}, i_{2}, j_{2}, \ldots, j_{s-1}, i_{s}, j_{s}, \ldots, i_{N-1}, j_{N-1}, i_{N}, j_{N}=1\right), \\
N \geq 1, \quad i_{s}, j_{s} \in\{1, \ldots, n\},
\end{gathered}
$$

удовлетворяющих условиям

$$
p\left(i_{s}, j_{s-1}\right) p\left(i_{s}, j_{S}\right)>0 \quad \forall s \in\{1, \ldots, N\}
$$

Для любой последовательности вида (3) определим следующее множество элементов группы $G$ :

$$
\begin{gathered}
\Omega(z)=\Omega\left(\xi_{i_{1}, 1}\right)^{-1} * \Omega\left(\xi_{i_{1}, j_{1}}\right) * \ldots * \Omega\left(\xi_{i_{s}, j_{s}-1}\right)^{-1} * \Omega\left(\xi_{i_{s}, j_{s}}\right) * \ldots \\
\ldots * \Omega\left(\xi_{i_{N}, j_{N-1}}\right)^{-1} * \Omega\left(\xi_{i_{N}, 1}\right)
\end{gathered}
$$

Для любых последовательностей $z_{1}, z_{2} \in Z$ рассмотрим последовательность $z \in Z$, полученную путем удаления из $z_{2}$ первого элемента, равного 1 , 
и приписывания результата к $z_{1}$ справа. Имеет место равенство множеств $\Omega(z)=\Omega\left(z_{1}\right) * \Omega\left(z_{2}\right)$. Следовательно, объединение множеств

$$
H=\bigcup_{z \in Z} \Omega(z)
$$

замкнуто относительно групповой операции и поэтому является подгруппой конечной группы $G$.

Используя введенные обозначения, сформулируем основной результат настоящей работы.

Теорема. 1) Справедливо неравенство

$$
\varepsilon_{N} \leq \sqrt{n}\left\|\bar{p}_{0}\right\| \lambda^{N} \quad \forall N \geq 1
$$

2) Если $P$ - вполне неразложимая дважды стохастическая матрица u группа подстановок $q(H)$ транзитивна на множестве $S$, то

$$
0 \leq \lambda<1
$$

Доказательство. 1) Пусть вектор начальных вероятностей цепи Маркова $\left\{\alpha_{N}\right\}_{N \geq 1}$ имеет вид

$$
\begin{gathered}
\bar{p}_{0}=\left(p_{0}(1), p_{0}(2), \ldots, p_{0}(n)\right), \quad p_{0}(1)+\ldots+p_{0}(n)=1 \\
p_{0}(i) \geq 0 \quad \forall i \in\{1, \ldots, n\} .
\end{gathered}
$$

Для любого представления $U \in \widehat{\mathrm{G}}^{(q)}$ степени $n_{U}$ определим следующие блочные матрицы $A(U), B(U)$ размеров $n_{U} \times\left(n n_{U}\right),\left(n n_{U}\right) \times n_{U}$ соответственно:

$$
\begin{aligned}
& A(U)=\left[\begin{array}{llll}
p_{0}(1) E_{n_{U}} & p_{0}(2) E_{n_{U}} & \ldots & p_{0}(n) E_{n_{U}}
\end{array}\right], \\
& B(U)=\left[\begin{array}{c}
E_{n_{U}} \\
E_{n_{U}} \\
\vdots \\
E_{n_{U}}
\end{array}\right],
\end{aligned}
$$

где $E_{n_{U}}-$ единичная квадратная матрица порядка $n_{U}$. Покажем, что для любых $N \geq 1, U \in \widehat{\mathrm{G}}^{(q)}$ имеет место равенство

$$
\Phi_{\eta^{(N)}}(U)=A(U) \Phi(U)^{N} B(U)
$$


Для любой последовательности

$$
l_{1}, l_{2}, \ldots, l_{N}, l_{N+1} \in\{1, \ldots, n\}
$$

условное распределение случайного элемента $\eta^{(N)}$ при условии

$$
\alpha_{1}=l_{1}, \alpha_{2}=l_{2}, \ldots, \alpha_{N}=l_{N}, \alpha_{N+1}=l_{N+1},
$$

совпадает с распределением произведения независимых случайных элементов

$$
\xi_{l_{1}, l_{2}}^{(1)} * \xi_{l_{2}, l_{3}}^{(2)} * \ldots * \xi_{l_{N}, l_{N+1}}^{(N)} .
$$

Из формулы полной вероятности и теоремы умножения для матричных характеристических функций (см. [1]) следует равенство

$$
\begin{aligned}
& \Phi_{\eta^{(N)}}(U)=\sum_{l_{1}, \ldots, l_{N+1}=1}^{n} p_{0}\left(l_{1}\right) p\left(l_{1}, l_{2}\right) \Phi_{\xi_{l_{1}, l_{2}}^{(1)}}(U) \times \\
& \times p\left(l_{2}, l_{3}\right) \Phi_{\xi_{l_{2}, l_{3}}^{(2)}}(U) \ldots p\left(l_{N}, l_{N+1}\right) \Phi_{\xi_{l_{N}, l_{N+1}}^{(N)}}(U) .
\end{aligned}
$$

Правая часть последнего равенства, в соответствии с соотношением (1) и правилами умножения блочных матриц, совпадает с правой частью равенства (6).

Для любых $N \geq 1, U \in \widehat{\mathrm{G}}^{(q)}$ из соотношения (6) и свойства мультипликативности эрмитовой нормы линейных отображений унитарных пространств следует неравенство

$$
\left\|\Phi_{\eta^{(N)}}(U)\right\| \leq\|A(U)\| \cdot\|\Phi(U)\|^{N} \cdot\|B(U)\| .
$$

Можно показать, что при любом $U \in \widehat{\mathrm{G}}^{(q)}$

$$
\|A(U)\|=\left\|\bar{p}_{0}\right\|, \quad\|B(U)\|=n^{\frac{1}{2}} .
$$

Обозначим через $k_{U}$ кратность неприводимой компоненты $U \in \widehat{\mathrm{G}}^{(q)}$ представления $Q$. Доказываемое соотношение (4) следует из соотношений (7), (2), (8), равенства для степени представления $Q$

$$
m=\sum_{U \in \widehat{\mathbb{G}}^{(q)}} n_{U} k_{U},
$$


и следующего неравенства, доказанного в [4]:

$$
\varepsilon_{N}^{2} \leq \frac{1}{m} \sum_{U \in \widehat{\mathbf{G}}^{(q)}, U \neq U_{0}} n_{U} k_{U}\left\|\Phi_{\eta^{(N)}}(U)\right\|^{2}
$$

2) Для любой комплексной матрицы $C$ обозначим через $C^{\top}$ транспонированную матрицу к матрице $C$ и для любого представления $U \in \widehat{\mathrm{G}}^{(q)}$ обозначим через $\Phi(U)^{*}$ сопряженную матрицу к матрице $\Phi(U)$, где

$$
\Phi(U)^{*}=\overline{\Phi(U)}^{\top}
$$

черта сверху обозначает взятие комплексно сопряженных элементов к элементам матрицы. Известно (см., например, [5]), что величина $\|\Phi(U)\|^{2}$ равна максимальному из модулей собственных чисел матрицы

$$
\Lambda(U)=\Phi(U)^{*} \Phi(U)
$$

Следовательно, для доказательства неравенства (5) достаточно показать, что для любого представления $U \in \widehat{\mathrm{G}}^{(q)}, U \neq U_{0}$, все собственные числа матрицы $\Lambda(U)$ по модулю строго меньше 1.

Введем матрицу $R=P^{\top} P=[r(i, j)]$. Из условия пункта 2) теоремы на матрицу $P$ и результатов работы [3] следует, что $R-$ (вполне) неразложимая дважды стохастическая матрица.

Для любых $i, j \in\{1, \ldots, n\}$, удовлетворяющих условию $r(i, j)>0$, рассмотрим непустое множество

$$
L_{i, j}=\{l \in\{1, \ldots, n\}: p(l, i) p(l, j)>0\}
$$

а также случайный элемент $\zeta_{i, j}$ со значениями в группе $G$ с распределением

$$
\mathrm{P}\left(\zeta_{i, j}=\sigma\right)=\frac{1}{r(i, j)} \sum_{l \in L_{i, j}} p(l, i) p(l, j) \mathrm{P}\left(\left(\xi_{l, i}^{(1)}\right)^{-1} * \xi_{l, j}^{(2)}=\sigma\right) \quad \forall \sigma \in G
$$

и носителем распределения

$$
\Omega\left(\zeta_{i, j}\right)=\bigcup_{l \in L_{i, j}}\left(\Omega\left(\xi_{l, i}\right)^{-1} * \Omega\left(\xi_{l, j}\right)\right)
$$


Из соотношений (1), (10), свойства унитарности представлений $U$ и теоремы умножения для матричных характеристических функций для любых $i, j \in$ $\in\{1, \ldots, n\}$ и $U \in \widehat{G}^{(q)}$ следуют равенства

$$
r(i, j) \Phi_{\zeta_{i, j}}(U)=\sum_{l=1}^{n} p(l, i) \Phi_{\xi_{l, i}}(U)^{*} p(l, j) \Phi_{\xi_{l, j}}(U)=\sum_{l=1}^{n} \Phi_{l, i}(U)^{*} \Phi_{l, j}(U) .
$$

Вследствие соотношений $(9)$ и (12) матрица $\Lambda(U)$ является блочной матрицей

$$
\Lambda(U)=\left[\begin{array}{cccc}
\Lambda_{1,1}(U) & \Lambda_{1,2}(U) & \ldots & \Lambda_{1, n}(U) \\
\Lambda_{2,1}(U) & \Lambda_{2,2}(U) & \ldots & \Lambda_{2, n}(U) \\
\vdots & \vdots & \vdots & \vdots \\
\Lambda_{n, 1}(U) & \Lambda_{n, 2}(U) & \ldots & \Lambda_{n, n}(U)
\end{array}\right]
$$

с $n^{2}$ квадратными блоками порядка $n_{U}$, у которой для любых $i, j \in\{1, \ldots, n\}$ на месте $i, j$ стоит блок

$$
\Lambda_{i, j}(U)=r(i, j) \Phi_{\zeta_{i, j}}(U)
$$

Необходимые и достаточные условия существования у таких блочных матриц собственных чисел, по модулю равных 1, получены автором в лемме из 3 работы [1]. Эти условия сформулированы в терминах свойств некоторой подгруппы группы $G$, которая, в свою очередь, описывается в терминах ненулевых элементов матрицы $R$ и множеств $\Omega\left(\zeta_{i, j}\right)$ для $i, j \in\{1, \ldots, n\}$, удовлетворяющих неравенству $r(i, j)>0$. Используя равенства (11), можно показать, что в нашем случае эта подгруппа совпадает с определенной выше подгруппой $H$.

Таким образом, по лемме из 33 работы [1] для любого представления $U \in \widehat{G}^{(q)}$ матрица $\Lambda(U)$ имеет собственное число, по модулю большее или равное 1 , тогда и только тогда, когда $U$ является неприводимой компонентой индуцированного представления группы $G$ единичным представлением ее подгруппы $H$. Это условие по теореме взаимности Фробениуса равносильно наличию единичной неприводимой компоненты у ограничения представления $U$ на подгруппу $H$. Следовательно, количество представлений $U \in \widehat{\mathrm{G}}^{(q)}$ с рассматриваемым свойством матрицы $\Lambda(U)$ не превосходит числа единичных компонент ограничения представления $Q$ на подгруппу $H$, т. е. числа орбит группы подстановок $q(H)$ на множестве $S$. Это число равно единице, так как по условию 2) формулировки теоремы группа подстановок $q(H)$ транзитивна на множестве $S$. Итак, матрица $\Lambda(U)$ может иметь собственное число, 
по модулю большее или равное 1 , не более чем для одного представления $U \in \widehat{\mathrm{G}}^{(q)}$.

С другой стороны, единичное представление $U_{0} \in \widehat{G}^{(q)}$, а матрица $\Lambda\left(U_{0}\right)=R$ имеет собственное число, равное 1. Следовательно, для любого представления $U \in \widehat{\mathrm{G}}^{(q)}, U \neq U_{0}$, все собственные числа матрицы $\Lambda(U)$ строго меньше 1. Согласно соотношению (2), имеет место двойное неравенство (5). Теорема доказана.

Приведем условия, при выполнении которых проверка соотношения (5) несколько упрощается. Предположим, что имеет место соотношение

$$
\bigcap_{j \in\{1, \ldots, n\}: p(i, j)>0} \Omega\left(\xi_{i, j}\right) \neq \oslash \quad \forall i \in\{1, \ldots, n\} .
$$

В частности, условие (13) выполняется в случае, когда распределения сомножителей в произведениях $\eta^{(N)}$ определяются состояниями (а не переходами) цепи Маркова $\left\{\alpha_{N}\right\}_{N \geq 1}$, т. е. при выполнении соотношений

$$
\xi_{i, j}=\xi_{i} \quad \forall i, j \in\{1, \ldots, n\}
$$

для некоторых случайных элементов $\xi_{1}, \ldots, \xi_{n}$ со значениями в группе $G$.

Зафиксируем произвольные элементы

$$
\sigma_{i, j} \in \Omega\left(\xi_{i, j}\right) \quad \forall i, j \in\{1, \ldots, n\}
$$

и рассмотрим следующее множество элементов группы $G$ :

$$
\Omega=\bigcup_{i, j \in\{1, \ldots, n\}: p(i, j)>0} \sigma_{i, j}^{-1} * \Omega\left(\xi_{i, j}\right) .
$$

Следствие. Если $P$ - вполне неразложимая дважды стохастическая матрица и множество подстановок $q(\Omega)$ порождает транзитивную группу подстановок на множестве S, то верно неравенство (5).

Доказательство. Согласно п. 2) теоремы достаточно показать, что группа подстановок $q(H)$ транзитивна на множестве $S$. Как уже упоминалось, $R-$ вполне неразложимая дважды стохастическая матрица. Из соотношений (11) и (13) следует, что

$$
e_{G} \in \Omega\left(\zeta_{i, j}\right) \quad \forall i, j \in\{1, \ldots, n\}: r(i, j)>0 .
$$


Из результатов работы [6] следует, что при этих условиях множество

$$
\bigcup_{i, j \in\{1, \ldots, n\}: r(i, j)>0} \Omega\left(\zeta_{i, j}\right)
$$

является системой порождающих элементов подгруппы $H$ группы $G$. Однако при любом $j \in\{1, \ldots, n\}$ имеет место неравенство $r(j, j)>0$, и, согласно соотношению (11),

$$
\sigma_{i, j}^{-1} * \Omega\left(\xi_{i, j}\right) \subseteq \Omega\left(\zeta_{j, j}\right) \quad \forall i, j \in\{1, \ldots, n\}: p(i, j)>0 .
$$

Следовательно, $\Omega \subseteq H$, и по условию следствия группа подстановок $q(H)$ транзитивна на множестве $S$. Следствие доказано.

\section{Список литературы}

1. Горчинский Ю.Н., Круглов И.А., Капитонов В.М. Вопросы теории распределений на конечных группах // В сб.: Труды по дискретной математике, т. 1. - 1997. - С. 85-112.

2. Круглов И.А. Оценка среднеквадратического уклонения от равновероятной матрицы для матриц переходных вероятностей произведений случайных величин со значениями в конечных группах, распределения которых определяются цепью Маркова // Обозр. прикл. и промышл. матем.2006. - Т. 13. Вып. 3. - С. 507-509.

3. Сачков В.Н. Вероятностные преобразователи и правильные мультиграфы.1 // В сб.: Труды по дискретной математике, т. 1. - 1997. C. $227-250$.

4. Горчинский Ю.Н., Капитонов В.М. О средних квадратических уклонениях в строках матриц переходных вероятностей на конечных группах подстановок // В сб.: Труды по дискретной математике, т. 2. - 1998. C. $88-100$.

5. Гантмахер Ф. Р. Теория матриц. - М.: Наука, 1988.

6. Круглов И.А. Принцип сходимости Б. М. Клосса для произведений случайных величин со значениями в компактной группе, распределения которых определяются цепью Маркова // Дискретная математика. - 2008. т. 20. Вып. 1. - С. 38-51. 